\title{
Téoros
}

Revue de recherche en tourisme

\section{La villégiature privée sur le territoire de la MRC d'Antoine-Labelle}

\section{Laval Dubois}

Volume 7, numéro 2, juillet 1988

Villégiature et tourisme

URI : https://id.erudit.org/iderudit/1080411ar

DOI : https://doi.org/10.7202/1080411ar

Aller au sommaire du numéro

Éditeur(s)

Université du Québec à Montréal

ISSN

0712-8657 (imprimé)

1923-2705 (numérique)

Découvrir la revue

Citer cet article

Dubois, L. (1988). La villégiature privée sur le territoire de la MRC

d'Antoine-Labelle. Téoros, 7(2), 20-22. https://doi.org/10.7202/1080411ar d'utilisation que vous pouvez consulter en ligne.

https://apropos.erudit.org/fr/usagers/politique-dutilisation/ 
La MRC d'Antoine-Labelle se situe dans les Hautes-Laurentides, soit à 208 kilomètres de Hull et à 238 kilometres de Montréal. Ce territoire a une superficie de $15801 \mathrm{~km}^{2}$, dont $4615 \mathrm{~km}^{2}$ sont constitués par des territoires non municipalisés. La géographie du territoire est dominée par une succession de collines entrecoupées par des vallées plus ou moins larges où l'on retrouve un réseau hydrographique bien hiérarchisé.

Les cours d'eau les plus importants, orientés nord-sud, sont la rivière Rouge, située à l'est et la rivière du Lièvre, située à l'ouest. Celles-ci ont constitué et constituent encore les axes dans lesquels se sont implantés les réseaux majeurs de communication tels le chemin de fer et les routes permettant de rejoindre la plaine du Saint-Laurent et la vallée de l'Outaouais.

Du point de vue juridique, la MRC d'Antoine-Labelle est constituée de 23 municipalités ayant une population totale de plus de 30000 personnes principalement localisées dans les vallées de la Lièvre et de la Rouge.

\section{La répartition temporelle et spatiale}

Le territoire de la MRC d'Antoine-Labelle fut ouvert a la colonisation il y a plus de 100 ans et, en raison des eléments naturels qu 'offrait la région, le tourisme et, par conséquent la villégiature, s'est implantée très tôt. Ainsi, la construction du chemin de fer et son prolongement jusqu’à Mont-Laurier (1909) a vraiment ouvert la région à la villégiature. Jusqu’à la construction de la route Montréal-Abitibi (1930-1940), le chemin de fer a constitué le facteur de localisation et la plupart des lacs à proximité ont été colonisés par la villegiature. A titre d'exemple, nous pouvons citer les lacs Nominingue et Gauvin où la villégiature s'est implantée en méme temps que le chemin de fer.

La construction de la route reliant Montréal à l'Abitibi ainsi que des routes secondaires vers l'intérieur ont rendu accessibles de nombreux lacs. Les années cinquante marquent le début d'une expansion rapide de la villégiature; ceci relié à une amélioration du réseau routier majeur (route 117) et secondaire et des facteurs sociaux tels l'industrialisation du Québec, l'exode rural et des heures de travail moins longues. Ce développement de la villégiature principalement axée sur le milieu aquatique, a été très important en bordure des lacs où la tenure du sol était privée, contrairement aux plans d'eau situés sur les terres du domaine public où l'implantation s'est faite plus tard. Il faut se rappeler que plus de $85 \%$ du territoire municipalisé est formé de terres du domaine public.

Ainsi, la tenure des bordures riveraines, dans le cas de la MRC d'Antoine-Labelle, a eu un impact quant à l'aspect temporel de l'implantation et Egalement à la densité d'occupation. Contrairement aux terres privées où les promoteurs tendaient à maximiser le nombre de terrains lotissables, le ministère, responsable du territoire public, avait déjà des normes quant aux superficies minimales, d'où un milieu de villégiature beaucoup plus aćré. Aujourd'hui, le territoire municipalisé de la MRC d'Antóine-Labelle compte plus de 8400 chalets répartis inégalement dans l'ensemble des municipalités en raison du nombre de plans d'eau, leur grandeur, l'accessibilité et la proximité des grandes artères de circulation.

L'implantation de la villégiature depuis ses débuts, à l'aube du XXe siècle, présente trois phases:
Début du XXe siècle - 1960: l'expansion Avec l'amélioration et l'ouverture de routes secondaires, la villégiature a été en expansion constante. L'offre de terrains disponibles comblait et même était supérieure à la demande.

1960 à 1980: la consolidation

L'ouverture continue de nouveaux plans d'eau tire à sa fin: l'on assiste à une consolidation des plans d'eau les plus accessibles. Cette période marque le début de la transformation de chalets en véritable résidenoes secondaires, des mutations résidentielles et de la construction de résidences principales en bordure des lacs a proximité des agglomérations.

Début 1980: la stabilisation

Depuis le début de cette décennie, l'expansion de la villégiature a considérablement ralentie. La crise économique du début de la décennie, l'entrée en vigueur de lois cadres, une planification municipale plus rigoureuse ont freiné le développement qui fut souvent effectué de façon anarchique. Depuis, les milieux de villégiature près des centres de services et des réseaux routiers ont subi des transformations soutenues axces surtout sur la mutation résidentielle et la construction de résidences permanentes. Quant aux résidences secondaires, l'expansion est très faible. Les nouvelles constructions, pour la plupart, sont des résidenoes secondaires au vrai sens du terme. Ces facteurs amenent la transformation de ces centres de villégiature en banlieue linéaire dont les résidents travaillent dans les centres urbains.

Bien que la villégiature ait connu une croissance dans la plupart des municipalites, certaines, comme nous le mentionnions précédemment, ont connu de très fortes augmentations en raison de la bonification de certains facteurs de localisation au détriment de d'autres municipalités où certains éléments diminuaient l'attrait, tels la distance par rapport aux axes routiers majeurs et même par rapport aux grands bassins de population (région montréalaise; région de la capitale nationale).

\section{La provenance du villégiateur}

La MRC d'Antoine-Labelle, de par sa situation et en raison des voles de communication importantes, constitue la limite nord de la zone récréative de la région montréalaise. Ainsi, plus de $64 \%$ des propriétaires de résidences secondaires proviennent de la région
- Monsieur Laval Dubois est coordonnateur a lamenatrise en getographie de I'Université de Sherbrooke. 
métropolitaine, $10 \%$ de la région de l'Outaouais québécois et ontarien, $20 \%$ par des résidents de la MRC d'Antoine-Labelle et $6 \%$ pour le reste du Québec, les autres provinces et les Etats-Unis.

Associé à la provenance du villégiateur, nous découvrons un élément de localisation: plus la municipalité se situe au nord, plus le nombre de propriétaires résidents dans une municipalité voisine augmente. Ainsi, la portion de propriétaires non résidants dans les municipalités de La Macaza et de Lac Nominingue situées au sud est de $90 \%$ et $95 \%$ tandis que dans la paroisse de Ferme-Neuve et Mont-Saint-Michel au nord, nous en retrouvons respectivement $47 \%$ et $51 \%$.

De façon générale, plus nous nous éloignons de la limite sud-est de la MRC, plus le nombre de propriétaires non résidents diminue.

À l'étude de la provenance, nous avons constaté que la région outaouaise était faiblement représentée et se concentrait dans quelques municipalités situées au sud-ouest, dans l'axe de la route 309 . Contrairement à l'axe de circulation Mont-Laurier-Montréal, I'axe Mont-Laurier-rivière Outaouais fut, jusqu'à récemment, de piètre qualité et la route est entièrement pavée depuis moins de 10 ans. Ainsi, le réseau routier fut un obstacle majeur à une forte présence de la population outaouaise au nord de Notre-Dame-du-Laus.

\section{Les caractéristiques socio- économiques du villégiateur}

Le type d'emploi du villégiateur montre qu'il existe une ventilation où les groupes les plus importants se situent parmi les ouvriers spécialises (18\%) et les professionnels (15\%). Également, les rentiers et ménagères possèdent $23 \%$ du stock de résidences secondaires. À l'exception de certaines inégalités, cette répartition se rapproche de la moyenne québécoise.

La répartition des emplois des villégiateurs de la MRC d'Antoine-Labelle montre que toutes les catégories d'emploi ont un accès à la possession d'une résidence secondaire, contrairement à la région de MagogOrford $^{(1)}$ où l'emploi semble constituer un facteur discriminant.

Quant au choix de la région, les motivations sont majoritairement la présence de liens familiaux et d'amis et la qualité du paysage. La distance et les facilités d'accès ne constituent pas un élément d'importance. Seulement $5 \%$ des villégiateurs les considèrent important contrairement à la région de Magog-Orford ${ }^{(2)}$ ou plus de $37 \%$ des répondants ont mentionné ce facteur. Ainsi, la MRC d'Antoine-Labelle, même en étant éloignée du bassin métropolitain (plus de 200 kilomètres) d'où provient plus de $60 \%$ de sa clientèle, exerce un attrait supérieur au désavantage que constitue la distance à parcourir.

La durée de séjour des villégiateurs est en moyenne de 35 jours, ce qui est semblable à la moyenne québécoise ${ }^{(3)}$, mais inférieure à la région de Magog-Orford. L'utilisation de la résidence secondaire est principalement axée sur le milieu aquatique; elle sert donc surtout pendant la saison estivale, contrairement aux régions plus au sud, où I'on retrouve des infrastructures de sports d'hiver (centre de ski) qui permettent une utilisation presque continue de la résidence secondaire. La MRC d'Antoine-Labelle a très peu de ces infrastructures, d'où une faible fréquentation pendant la saison hivernale. D'ailleurs, les activités des villégiateurs sont majoritairement reliées à l'élément aquatique (voile $70 \%$; plage-natation $94 \%$; peche $64 \%$ ), ce qui est supérieur aux données recueillies pour l'ensemble du Québec ${ }^{(4)}$.

La valeur moyenne de la résidence secondaire sur le territoire de la MRC d'AntoineLabelle est de $121^{166 \$^{(5)}}$. Ventilées par municipalité, les valeurs adoptent un modèle concentrique, c'est-à-dire que celles-ci diminuent progressivement vers les municipalités les plus septentrionales. Par ailleurs, ce même modèle $s^{*}$ applique également pour la répartition résidents - non-résidents. Ceci implique que plus il y a de résidents propriétaires de résidences secondaires à la MRC, plus les valeurs moyennes diminuent.

Sans porter un jugement de valeur, ces variations spatiales pourraient être liées d'une part à des motivations du non-résident qui aspire au calme de la nature en opposition à la vie trépidante de la ville tandis que pour le résident, le chalet constitue un lieu de transition puisqu'il est déjà en contact avec les grands espaces et la campagne. De meme, les valeurs supérieures des résidences secondaires des non-résidents sont souvent liées à une installation definitive lors de leur retraite d'où une résidence secondaire de plus grande superficie, mieux isolée, etc. de même qu'un terrain généralement plus grand.

\section{Économie, services municipaux, pouvoir politique}

La villégiature, en terme de retombees économiques, injecte dans la région plus de $7000000 \$^{(6)}$ sans compter les taxes foncières. À cet égard, $24 \%$ de l'assiette fiscale de la MRC provient de la résidence secondaire avec des maxima de plus de $46 \%$ pour certaines municipalités. À l'exception des municipalités de ville ou village, la villégiature constitue, dans le budget municipal, un apport très important. Le TFR ${ }^{(7)}$ (taux de fonction résidentielle) permet de visualiser l'impact économique de la villégiature. Sur le territoire, le TFR se situe à $165 \%$ avec des maxima de $364 \%$ (municipalités au sud) et des minima de $0.069 \%$ (municipalités de village). De plus, ce TFR est beaucoup plus bas dans les municipalités septentrionales où l'économie s'appuie davantage sur d'autres ressources telles l'agriculture, la forêt. Selon certains auteurs, un taux excédant $60 \%$ implique une dépendance économique face à l'activité que constitue la villégiature et, par conséquent, l'économie reliếe au tourisme.
La villégiature, dans le passé, constituait un apport économique net, en ce sens que la municipalité ne donnait aucun service. Depuis quelques années, suite aux transformations qu'a subi la villégiature traditionnelle, les municipalités sont confrontées à une problématique relice aux services que ces villégiateurs et résidents demandent avec insistance. D'une occupation saisonnière, cette activité est devenue annuelle et nécessite de la part de la municpalité l'ouverture de ces chemins pendant la période hivernale. Cette problématique des chemins de villégiature est reliće au fait que dans plusieurs cas, ce ne sont que des droits de passages ou chemins privés dont les dimensions ne sont pas conformes. Enfin, ces nouveaux milieux sédentarisés demandent des services (ex.: les loisirs); il en résulte un éclatement du noyau historique qui, d'espace central, perd ses facteurs de localisation au profit de nouveaux noyaux.

Le villégiateur, jusqu'à récemment, n'a jamais joué un rôle politique important. Avec l'émergence d'associations de lacs et d'une conscience plus aigüe de la qualité de l'environnement, le villégiateur s'implique aujourd'hui avec beaucoup plus de vigueur. Sa vision s'aligne essentiellement vers une protection accrue de l'environnement tandis. que le résident doit adapter cet environnement. Ces positions opposées se répercutent au conseil municipal. Face à ces situations où le villégiateur considère que ses opinions ne sont pas retenues, l'action politique directe (élection) permet d'influer sur les décisions des élus municipaux.

Par l'action conjuguée, de la force fiscale des propriétaires de résidences secondaires et de la sédentarisation de certains d'entre eux, le pouvoir n'est plus exercé par les seuls résidents permanents dans bon nombre de municipalités. Or, les intérêts de ceux-ci et de ceux-là ne sont pas nécessairement concordants $^{(8)}$. Bien que cette situation ne soit pas vécue avec autant d'intensité ici que dans les basses Laurentides, plusieurs municipalités où le nombre de villégiateurs est important, vivent cette réalité. Ainsi, une orientation municipale, qui s'avère très environnementaliste, peut mener dans les municipalités rurales à une dépendance encore plus grande envers l'économie reliée au tourisme. Ceci favorise surtout un tertiaire induit et non pas un tertiaire inducteur, c'est-ă-dire aucunement structurant ${ }^{(9)}$

\section{La villégiature et ses perspectives}

À l'expansion soutenue des dernières décennies, nous assistons depuis le début de cellesci, à un net ralentissement de l'implantation de résidences secondaires. De 1961 à 1980 , l'augmentation moyenne annuelle fut de $8,5 \%$; depuis 1980 , cette augmentation se situe à $0,24 \%$. La crise économique du début de la décennie, l'entrée en vigueur de loi cadre sur l'aménagement, une réglementation municipale plus structurée, des normes environnementales plus sévères ainsi que les 


\begin{tabular}{|c|c|c|c|c|c|c|c|c|c|}
\hline \multirow{2}{*}{ Municipalités } & \multirow[t]{2}{*}{$\begin{array}{l}\text { Superficie } \\
\text { km2 }\end{array}$} & \multirow[t]{2}{*}{$\begin{array}{l}\text { Population } \\
1986\end{array}$} & \multicolumn{3}{|c|}{$\begin{array}{l}\text { Nombre de résidences } \\
\text { secondaires }\end{array}$} & \multicolumn{2}{|c|}{$\begin{array}{l}\text { Propriété de } \\
\text { la résidence } \\
\text { secondaire } 1986\end{array}$} & \multirow{2}{*}{$\begin{array}{l}\text { TFR(1) } \\
1986 \\
\%\end{array}$} & \multirow{2}{*}{$\begin{array}{l}\text { Ev. rés. } \\
\text { sec. } \\
\text { Ev. tot. } \\
1986 \\
\%\end{array}$} \\
\hline & & & .61 & 80 & 86 & $\begin{array}{l}\text { Rés. } \\
\%\end{array}$ & $\begin{array}{l}\text { Non res. } \\
\%\end{array}$ & & \\
\hline Beaux-Rivages & 143 & 978 & 155 & 403 & 409 & 34,5 & 65,5 & 178 & 28 \\
\hline Chute-St-Philippe & 239 & 602 & 130 & 536 & 530 & 15,5 & 84,7 & 305 & 46 \\
\hline Des Ruisseaux & 508 & 3889 & 287 & 555 & 561 & 52,6 & 47,4 & 56 & 10 \\
\hline Ferme-Neuve, paroisse & 1028 & 854 & 91 & 231 & 239 & 53 & 47 & 169 & 12 \\
\hline Ferme-Neuve, village & 3,1 & 2172 & 7 & 7 & 7 & 100 & - & 1 & 0,12 \\
\hline Kiamika & 258,5 & 591 & 134 & 219 & 221 & 26,6 & 73,4 & 166 & 25 \\
\hline Lac-des-Écorces & 4,4 & 847 & 1 & 2 & 3 & - & - & 0,01 & 0,25 \\
\hline Lac-du-Cerf & 78,4 & 371 & 127 & 354 & 360 & 13,9 & 86,1 & 302 & 43 \\
\hline Lac Nominingue & 197 & 1611 & 643 & 1163 & 1199 & 5 & 95 & 268 & 40 \\
\hline Lac St-Paul & 109,7 & 391 & 81 & 254 & 259 & 25,7 & 74,3 & 270 & 36 \\
\hline La Maciza & 161 & 863 & 166 & 747 & 735 & 10,7 & 89,3 & 364 & 46 \\
\hline L'Annonciation & 10,46 & 2318 & 7 & 7 & 6 & - & - & 0,069 & 0,3 \\
\hline L'Ascension & 309,42 & 626 & 121 & 512 & 525 & 12,5 & 87,5 & 336 & 42 \\
\hline Marchand & 180 & 1249 & 224 & 647 & 663 & 15,6 & 84,4 & 185 & 38 \\
\hline Mont-Laurier & 82 & 7937 & 45 & 95 & 94 & 48,8 & 41,2 & 2,7 & 12 \\
\hline Mont St-Michel & 137,6 & 680 & 59 & 146 & 149 & 49 & 51 & 97 & 18 \\
\hline Notre-Dame-Pontmain & 268 & 533 & 118 & 295 & 317 & 12,2 & 87,8 & 223 & 30 \\
\hline Notre-Dame-du-Laus & 889 & 1327 & 268 & 994 & 1023 & 9,8 & 90,1 & 293 & 37 \\
\hline Lae Saguay & 141 & 301 & 82 & 130 & 138 & 19,7 & 80,1 & 122 & 18 \\
\hline St-Aimé-Lac-des-Iles & 165,6 & 665 & 89 & 205 & 208 & 38 & 62 & 129 & 18 \\
\hline Ste-Anne-du-Lac & 180,9 & 582 & 56 & 144 & 154 & 34,5 & 65,5 & 83 & 12 \\
\hline Ste-Véronique & 166,6 & 945 & 185 & 427 & 437 & 17,4 & 82,6 & 160 & 25 \\
\hline Val-Barrette & 4,8 & 574 & 80 & 120 & 122 & 28,7 & 71,3 & 68 & 21 \\
\hline MRC d'Antoine-Labelle & & 30906 & 3156 & 8309 & 8434 & 20,5 & 79,5 & 165 & 24 \\
\hline
\end{tabular}

1: Taux fonction résidentielle $=$ Nombre de résidences secondaires $\times 100$ Nombre de résidences principales

Source: Răle d'évaluation, MRC d'Antoine-Labelle, 1986.

coûts des terrains et de construction, ont un effet marqué sur la construction de résidences secondaires.

Les mutations résidentielles constituent un second facteur dans les changements de la villégiature. Bien que ces mutations soient moins perceptibles que dans le sud du Québec, celles-ci sont présentes surtout dans les zones a proximité du réseau routier majeur et/ou à proximité des agglomérations. Ces changements sont reliés d'une part à la venue de retraités qui transforment leur résidence secondaire en résidence principale ainsi qu"à la présence de travailleurs demeurant dans ces zones de villégiature et travaillant dans les centres. Ce dernier élément affecte les plans d'eau situés près du centre régional et des sous-centres régionaux. Ce mouvement pendulaire, sans être de l'ampleur des régions situées en périphérie de Montréal, en est semblable.

A cette mutation résidentielle s'ajoute la construction de résidences permanentes (plus de $30 \%$ des nouvelles résidences en bordure de plans d'eau) qui changent encore plus le portrait des zones de villégiature. Ainsi, la villégiature sur le territoire de la MRC d'Antoine-Labelle connaît un ralentissement marqué d'où une stabilisation du nombre de résidences secondaires. Les facteurs liês à la mutation résidentielle et à la construction de résidences principales seront déterminants surtout pour les plans d'eau à proximité des réseaux routiers importants, des centres dispensateurs d'emploi et des centres de services. Quant aux autres plans d'eau, la vocation de villégiature se maintiendra; toutefois les bâtiments seront généralement des résidences secondaires au vrai sens du terme, impliquant une utilisation pouvant être répartie tout au long de l'année. $f$

Notes explicatives

(1) GILBERT, D., La villtgiature privede: application a la rógion de Magog-Orford. Département de gotographie, Universite de Sherbrooke, 1986, page 220.
2) Ibid.., p. 121.

(3) Ministerre du Loisir, de la Chasse et de la Pache, La place de la villégiatura privée dans les loisirs des Quóbecois et dans leur vie sociale et bconomique. Québec, 1983, page 24.

(4) Ibid., p. 37.

(5) MAC d'Antoine-Labelle, Roles d'dvaluation municipale 1986.

(6) CIDEL, L'impact du tourisme ot de la villégiature sur les commercents at les travailleurs du comté de Labolle, Mont-Laurier, 1981, page 93.

(7) TFR, Taux de fonction residentiblle: Nombre de residences socondaires $x 100$ Nombre de résidences principales

(8) SAMSON, M., La rósidence secondaira: wers une nouvelle classe de citoyons?. LRM, mars 1986, page 31 .

(9) ST-AMOUR, J.-P., La villégiature au Quábec, Edtion Asticou, Hull, 1979 , page 43. 